\title{
On the Performance of RIS-Assisted Dual-Hop UAV Communication Systems
}

\author{
Liang Yang, Fanxu Meng, Jiayi Zhang, Mazen O. Hasna, and Marco Di Renzo
}

\begin{abstract}
In this paper, to further reduce the bit error rate, and improve the coverage and reliability of unmanned aerial vehicle (UAV) communication systems, we propose a reconfigurable intelligent surface (RIS)-assisted UAV scheme, where the RF signal generator sends an unmodulated carrier signal to the RIS, then the RIS modulates each signal, and we use them as a transmitter. The UAV is applied as a relay to forward the information to the destination. In particular, we use a $k-\mu$ distribution to model the RIS-assisted ground-to-air (G2A) links. With this model, the analytical expressions of outage probability and average bit error rate (BER) are derived. Results show that the use of the RIS can effectively improve the coverage and reliability of the UAV communication systems.
\end{abstract}

Index Terms-UAV, RIS, $k-\mu$ distribution, outage probability, bit error rate.

\section{INTRODUCTION}

Unmanned Aerial Vehicle (UAV) communication is playing an increasingly important role in today's wireless communication systems. Due to their small size and flexibility, UAVs are widely used for rapid networking in communication-disabled areas. Also, UAVs can be used to network for areas where signals from the base station are difficult to reach, such as mountains and deep desert areas.

Research on UAV communication systems is gaining more and more attention. In [1], a UAV-aided relaying system with energy harvesting function was proposed, and the outage probability of different urban environmental parameters was analyzed. In [2], the secrecy performance of the air to air (A2A) communication system in the presence of eavesdroppers was studied, which shows that increasing the coverage of the UAV can enhance the average secrecy capacity. In [3], the authors analyzed the physical layer security of a UAV communication system in a Rician fading channel with a spatial Poisson distributed eavesdropper. In [4], the authors used the drone as a relay station and optimized the trajectory of the mobile UAV relay to achieve the maximum throughput.

Reconfigurable intelligent surfaces (RISs) are man-made surfaces of electromagnetic (EM) material that can be electronically controlled by integrated electronics. As an emerging technology, RISs have shown great potential in the communication field and are expected to become one of the key technologies of 6G. Recent results show that an RIS can effectively control the wavefront of an impact signal, such as the phase, amplitude, and frequency, without the need for complex decoding, encoding, and RF processing operations. In [5], the authors outlined current research findings and availability of intelligent reconfigurable meta-surfaces. In [6], the authors gave a detailed overview and historical review of the latest solutions of RISs, expounded the differences between the RIS and other technologies, and the current problems to be solved, and rethought the communication theoretical model in wireless networks. In [7], the authors used the RIS to improve the achievable rate of the UAV-enabled communication system and proposed an optimization algorithm to maximize the average achievable rate. In [8], a deep learning method was proposed to effectively configure the online wireless configuration of RISs in an indoor communication environment, which could effectively improve the achievable throughput of the target user. In [9], applying RISs to a downlink multi-antenna multiuser communication system was studied.

Different from the downlink transmission system proposed in [7] where the UAV is used as a transmitter and the RIS reflects the signal from the UAV to the user, we propose an RIS-assisted dual-hop UAV communication system where the RIS is used as a transmitter and the UAV is used as a relay to further enhance the height and reliability. In this system, we assume that the RIS is located very close to the radio frequency $(\mathrm{RF})$ signal generator and then the RIS along with the RF signal generator can be used as a transmitter to reflect the signal to the UAV through a ground-to-air (G2A) link. Notice that using RISs as a transmitter has been investigated in [6] and references there in. More specifically, if the RIS consists of $N$ reconfigurable meta-surfaces, and its reflection phases can be optimized independently of each other, then a $N$-stream virtual multiple input multiple output (MIMO) system can be realized by using a single RF active chain. Compared with the conventional MIMO system, using the RIS as a transmitter offers a unique advantage that very large antenna arrays can be implemented using with a few, possibly one, RF chain. Thus, the cost of using many RF chains in the traditional MIMO system can be reduced. In particular, the main contributions of this work include three parts. First, we use the $k-\mu$ distribution to model the channel fading for the RIS-assisted G2A link. Second, we present the analysis for the outage probability and average bit-error rate (BER) of the presented system. Finadly, the optimal altitude and position of the UAV are also investigated.

\section{System AND Channel MODELS}

As shown in Fig. 1, consider an RIS-assisted UAV communication system including an RF signal generator, an RIS, a UAV relay station, and one destination (D). In this system, we use the RIS and RF signal generator together as a transmitter (T) where the RIS is installed to very close to the RF signal generator, and hence the channel fading between them is 
negligible. Similar to most relaying systems, two time slots are needed to complete the information transmission. At the first stage, $\mathrm{T}$ sends a signal to the UAV through an RISassisted G2A link, where the RF signal generator sends an unmodulated carrier signal to the RIS, then the RIS modulates each signal and optimizes the phase to maximize the signalto-noise ratio (SNR) of the received signal at the UAV [6]. In the second stage, we use the decode and forward (DF) method and assume that the UAV can successfully decode the received signal. Then, the UAV sends the decoded signal to D through an air-to-ground (A2G) link.

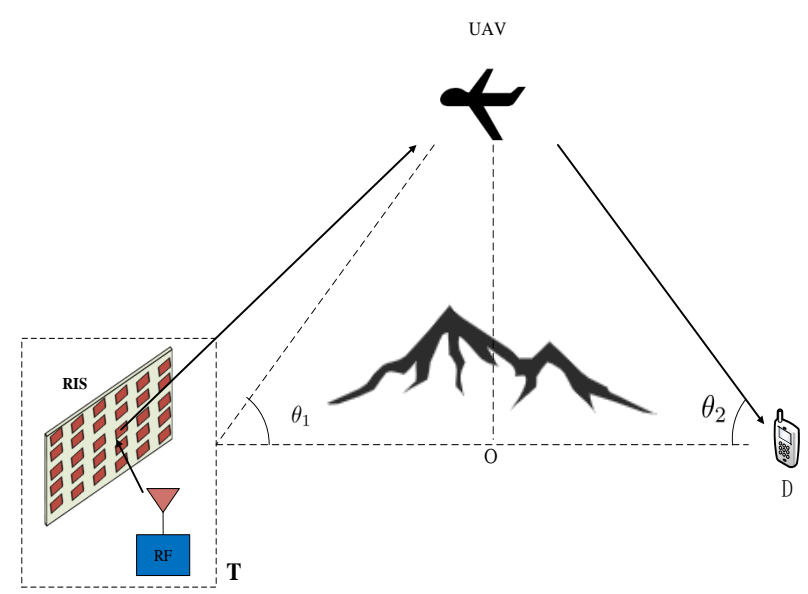

Fig. 1. System diagram of RIS-assisted UAV communication systems

\section{A. RIS-Assisted G2A Link}

In the first time slot, $\mathrm{T}$ sends the signal to the UAV through an RIS-assisted G2A link. First, the RF signal generator generates an unmodulated carrier signal $\cos \left(2 \pi f_{c} t\right)$ and sends it to the RIS. Then, the RIS performs the phase modulation to obtain a modulated signal. Moreover, in the process of the phase modulation, not only the information bits are modulated, but also the reflection phase is optimized to maximize the SNR of the received signal at the UAV. Therefore, the received signal at the UAV can be written as

$$
y_{U}=\left[\sum_{i=1}^{N} h_{i} e^{j\left(\varphi_{i}+w_{m}\right)}\right] \sqrt{P_{T}}+n_{1},
$$

where $h_{i}=\frac{1}{\sqrt{L_{T U}}} \beta_{i} e^{-\psi_{i}}$ is the channel gain, $L_{T U}=$ $10 \log 10\left(l_{T U}^{\alpha}\right)+A$ is the path loss, and $A$ is a constant determined by the signal frequency and transmission environment, and $l_{T U}=\sqrt{h^{2}+r_{T U}^{2}}$ is the distance between $\mathrm{T}$ and the $\mathrm{UAV}, h$ is the height of the UAV, $r_{T U}$ is the horizontal distance between $\mathrm{T}$ and the UAV, $\alpha$ is the path loss exponent, $P_{T}$ is the power of the unmodulated signal, $\varphi_{i}$ is the optimized phase induced by the $i$ th reflecting element of the RIS, $w_{m}$ is the modulation phase of the information carried by the RIS, and $n_{1} \sim \mathcal{C N}\left(0, N_{0}\right)$ is the additive white Gaussian noise (AWGN). From [6], we know that the maximum SNR can be obtained when $\varphi_{i}=\psi_{i}$. Thus, the received signal can be rewritten as

$$
y_{U}=\sqrt{\frac{P_{T}}{L_{T U}}}\left[\sum_{i=1}^{N} \beta_{i}\right] e^{j w_{m}}+n_{1} .
$$

From (2), the maximum instantaneous SNR at the UAV can be expressed as

$$
\gamma_{U}=\frac{\left(\sum_{i=1}^{N} \beta_{i}\right)^{2} P_{T}}{N_{0} L_{T U}}=\frac{R^{2} P_{T}}{N_{0} L_{T U}},
$$

where $R$ is the sum of $N$ independent and identically distributed (i.i.d.) Rician random variables (RVs) $\beta_{i}, i=1, \ldots, N$.

For the $\mathrm{G} 2 \mathrm{~A}$ or $\mathrm{A} 2 \mathrm{G}$ channels in UAV networks, the widely used model is the Rician fading model due to the existence of a line-of-sight ( $\mathrm{LoS}$ ) component. However, the G2A link considered in this work is unlike the ones in the literature since the RIS is involved. Thus, we can not simply apply the Rician model to characterize the statistical distribution of the G2A link. From [10], the physical model of the $k-\mu$ distribution considers that the signal is composed of multipath wave clusters propagating in the same environment, and each cluster has its own dominant wave and scattered wave components. Given that the sum of $N$ i.i.d. Rician RVs is a $k$ - $\mu$ distribution, we model $R$ as a $k$ - $\mu \mathrm{RV}$ with a probability density function (PDF) of $\gamma_{U}$ readily given by

$$
\begin{aligned}
f_{\gamma_{U}}\left(\gamma_{U}\right)= & \frac{\mu(1+k)^{\frac{\mu+1}{2}}}{k^{\frac{\mu-1}{2}} \Omega^{\frac{\mu+1}{2}}} \frac{\gamma_{U}^{\frac{\mu-1}{2}}}{\bar{\gamma}_{1}^{\frac{\mu+1}{2}}} \exp \left(\mu\left[-k-\frac{(1+k)}{\Omega} \frac{\gamma_{U}}{\bar{\gamma}_{1}}\right]\right) \\
& \times I_{\mu-1}\left(2 \mu \sqrt{\frac{k(1+k) \gamma_{U}}{\Omega \bar{\gamma}_{1}}}\right)
\end{aligned}
$$

where $\bar{\gamma}_{1}=\frac{P_{T}}{N_{0} L_{T U}}, \Omega=E\left(R^{2}\right), I_{\mu-1}(\cdot)$ denotes the modified Bessel function of the first class and $k \geq 0$ is the ratio between the total power of the dominant components and the total power of the scattered waves. From [11], the values of parameters $k$ and $\mu$ are given by

$$
\begin{gathered}
\mu=\frac{\Omega^{2}}{\left(E\left(R^{4}\right)-\Omega^{2}\right)} \frac{(1+2 k)}{(1+k)^{2}}, \\
k^{-1}=\frac{\sqrt{2}\left(E\left(R^{4}\right)-\Omega^{2}\right)}{\sqrt{2 E^{2}\left[R^{4}\right]-\Omega^{2} E\left[R^{4}\right]-\Omega E\left(R^{6}\right)}}-2,
\end{gathered}
$$

where $E\left(R^{2}\right), E\left(R^{4}\right)$ and $E\left(R^{6}\right)$ are the moments of $R$, respectively. From [10], $E\left(R^{2}\right), E\left(R^{4}\right)$ and $E\left(R^{6}\right)$ can be evaluated as

$$
\begin{aligned}
E\left[R^{n}\right] & =\sum_{n_{1}=0}^{n} \sum_{n_{2}=0}^{n_{1}} \ldots \sum_{n_{N-1}=0}^{n_{N-2}}\left(\begin{array}{c}
n \\
n_{1}
\end{array}\right)\left(\begin{array}{c}
n_{1} \\
n_{2}
\end{array}\right) \ldots\left(\begin{array}{c}
n_{N-2} \\
n_{N-1}
\end{array}\right) \\
& \times E\left[\beta_{1}^{n-n_{1}}\right] E\left[\beta_{2}^{n_{1}-n_{2}}\right] \ldots E\left[\beta_{N}^{n_{N-1}}\right]
\end{aligned}
$$

where $E\left[\beta_{i}^{n}\right]=\frac{\Gamma(1+n / 2) \exp \left(-K_{i}\right) \Omega_{i}^{n / 2}}{\left(1+K_{i}\right)^{n / 2}} F_{1}\left(1+\frac{n}{2} ; 1 ; K_{i}\right), \Omega_{i}=$ $E\left[\beta_{i}^{2}\right]$ is the mean power of $\beta_{i}, E\left[\beta_{i}^{n}\right]$ is the $n$th moment of $\beta_{i}, K_{i}$ is the Rician factor of $\beta_{i}, \Gamma(\cdot)$ is the gamma function and ${ }_{1} F_{1}(\cdot ; \cdot ; \cdot)$ is the confluent hypergeometric function. As mentioned earlier, the $k$ - $\mu$ distribution requires that $k$ is a 
non-negative constant, but computing $k$ by using (6) may not meet the requirement, which has been observed in [10]. Notice that the $k-\mu$ distribution reduces to the exact Nakagami-m distribution when $\mu=m$ and $k \rightarrow 0$.

\section{B. A2G Link}

In the second stage, the UAV sends the decoded signal to D through an A2G link. Then, the received signal at D can be expressed as

$$
y_{D}=g x+n_{2},
$$

where $g=\frac{1}{\sqrt{L_{U D}}} \beta_{U D} e^{\xi_{i}}$ is the channel gain of the A2G link, and $n_{2} \sim \mathcal{C N}\left(0, N_{0}\right)$ denotes the AWGN noise, and $x$ denotes the encoded signals. Therefore, the instantaneous SNR at D can be written as

$$
\gamma_{D}=\frac{\left|\beta_{U D}\right|^{2} P_{U}}{N_{0} L_{U D}},
$$

where $L_{U D}=10 \log 10\left(l_{U D}^{\alpha}\right)+A$ is the path loss, $P_{U}$ is the transmit power of the UAV, $l_{U D}=\sqrt{h^{2}+r_{U D}^{2}}$ is the distance between $\mathrm{D}$ and the $\mathrm{UAV}$, and $r_{U D}$ is the horizontal distance between UAV and D. From [12], the PDF of $\gamma_{D}$ can be written as

$$
\begin{aligned}
f_{\gamma_{D}}\left(\gamma_{D}\right)= & \frac{(1+K) e^{-K}}{\bar{\gamma}_{2}} \exp \left[-\frac{(1+K) \gamma_{D}}{\bar{\gamma}_{2}}\right] \\
& \times I_{0}\left(2 \sqrt{\frac{K(1+K) \gamma_{D}}{\bar{\gamma}_{2}}}\right),
\end{aligned}
$$

where $\bar{\gamma}_{2}=\frac{P_{U}}{N_{0} L_{U D}}, K$ is the Rician fading factor.

The value of the path loss is usually determined by the density of obstacles on the propagation path, so we model the path loss index with LoS probability [13] as $\alpha(\theta)=$ $a_{1} P_{L o S}(\theta)+b_{1}$, where $P_{L o S}$ is the LoS probability, $a_{1}$ and $a_{2}$ are determined by the environment and transmission frequency, $\theta \in\left\{\theta_{1}, \theta_{2}\right\}$, and $\theta_{1}$ and $\theta_{2}$ represent the angles between the UAV and T, D, respectively. Similarly, we model the Rician factor as a function of $\theta$. From [14], $K(\theta)=a_{2} \cdot e^{b_{2} \theta}$, where $a_{2}=k_{0}$ and $b_{2}=\frac{2}{\pi} \ln \left(\frac{k_{0.5 \pi}}{k_{0}}\right)$.

\section{Performance analysis}

In this section, using the above obtained statistical distributions, we analyze the outage probability and average BER, respectively.

\section{A. Outage Probability Analysis}

The reliability of a communication link is usually assessed by the outage probability. Since we use the DF protocol at the UAV, a successful communication requires both links to be uninterrupted. Thus, the outage probability can be readily expressed as

$$
\begin{aligned}
P_{\text {out }} & =\operatorname{Pr}\left(\min \left\{\gamma_{\mathrm{U}}, \gamma_{\mathrm{D}}\right\} \leq \gamma_{\mathrm{th}}\right) \\
& =1-\left(1-P_{T U}\right)\left(1-P_{U D}\right),
\end{aligned}
$$

from (3) and (4), $P_{T U}$ can be written as

$$
\begin{aligned}
P_{T U} & =\operatorname{Pr}\left(\gamma_{\mathrm{U}} \leq \gamma_{\mathrm{th}}\right) \\
& =1-Q_{\mu}\left(\sqrt{2 \mu k}, \sqrt{\frac{2 \mu \gamma_{t h}(1+k)}{\Omega \bar{\gamma}_{1}}}\right),
\end{aligned}
$$

where $Q_{\mu}(\cdot ; \cdot)$ is the Marcum Q-function with parameter $\mu$. Similarly, from (9) and (10), $P_{U D}$ can be obtained as

$$
\begin{aligned}
P_{U D} & =\operatorname{Pr}\left(\gamma_{\mathrm{D}} \leq \gamma_{\mathrm{th}}\right) \\
& =1-Q_{1}\left(\sqrt{2 K}, \sqrt{2 \gamma_{t h}(1+K) / \bar{\gamma}_{2}}\right),
\end{aligned}
$$

with (11), (12) and (13), we can write the overall outage probability as

$$
\begin{aligned}
P_{\text {out }}=1 & -Q_{1}\left(\sqrt{2 K}, \sqrt{2 \gamma_{t h}(1+K) / \bar{\gamma}_{2}}\right) \\
& \times Q_{\mu}\left(\sqrt{2 \mu k}, \sqrt{\frac{2 \mu \gamma_{t h}(1+k)}{\Omega \bar{\gamma}_{1}}}\right) .
\end{aligned}
$$

\section{B. Average Bit Error Rate}

The BER is a good measure of the accuracy of data transmission. For a dual-hop communication system with the DF protocol, the average BER can be written as [15]

$$
P_{B E R}=P_{E 1}+P_{E 2}-2 P_{E 1} P_{E 2},
$$

where $P_{E 1}$ and $P_{E 2}$ are the average BERs for the first and second hop, respectively. For different binary modulation schemes, the unified average BER expression is given by [16]

$$
P_{B E R}=\frac{q^{p}}{2 \Gamma(p)} \int_{0}^{\infty} \exp (-q \gamma) \gamma^{p-1} F(\gamma) d \gamma,
$$

where the parameters $p$ and $q$ denote different modulation schemes, such as $p=0.5$ and $q=1$ for binary phase shift keying (BPSK), $p=0.5$ and $q=0.5$ for binary frequency shift keying (BFSK), and $p=1$ and $q=1$ for differential phase shift keying (DPSK). In this work, we consider the DPSK modulation.

From [17, eq. (8)] and with (12) (16), $P_{E 1}$ can be exactly derived as

$$
P_{E 1}=\left(\frac{\mu(1+k)}{\mu(1+k)+\Omega \bar{\gamma}_{1}}\right)^{\mu} e^{-\mu k}{ }_{1} F_{1}\left(\mu ; \mu ; \frac{\mu^{2} k(1+k)}{\mu(1+k)+\Omega \bar{\gamma}_{1}}\right)
$$

Similarly, using (13) and (16), and [17, eq. (8)], we have

$$
P_{E 2}=\left(\frac{1+K}{1+K+\bar{\gamma}_{2}}\right) e^{-K}{ }_{1} F_{1}\left(1 ; 1 ; \frac{K(1+K)}{1+K+\bar{\gamma}_{2}}\right)
$$

\section{NUMERICAL RESULTS}

In this section, we present the numerical results to verify our analysis. The parameters used in the following figures are set to $f_{c}=1 \mathrm{GHz}, k_{0}=5 \mathrm{~dB}, k_{0.5 \pi}=15 \mathrm{~dB}, a_{1}=-1.5$, $b_{1}=3.5, p=1, q=1, \bar{\gamma}_{U}=P_{T} / N_{0}$ is the average SNR at the UAV, and $\rho=\frac{r_{T U}}{r_{T U}+r_{U D}}$ represents the horizontal distance ratio of the first link to the sum of two links. For the system without the RIS, we assume that the source with one antenna communicates directly with the UAV, and the channel fading follows the Rician distribution. Moreover, all simulation results are obtained in an urban environment. 


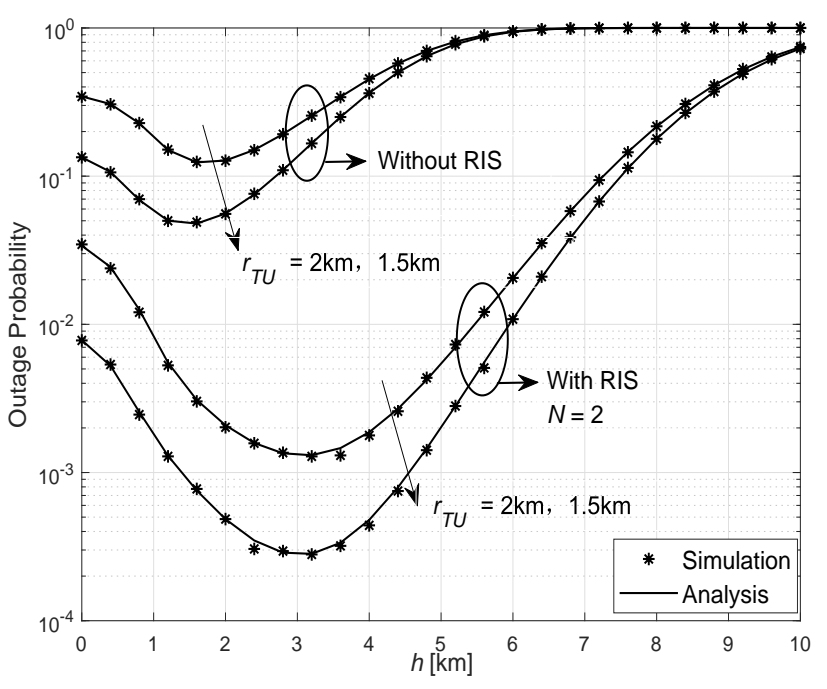

Fig. 2. Outage probability versus $h$ for dual-hop UAV systems with and without RISs for different coverage radius

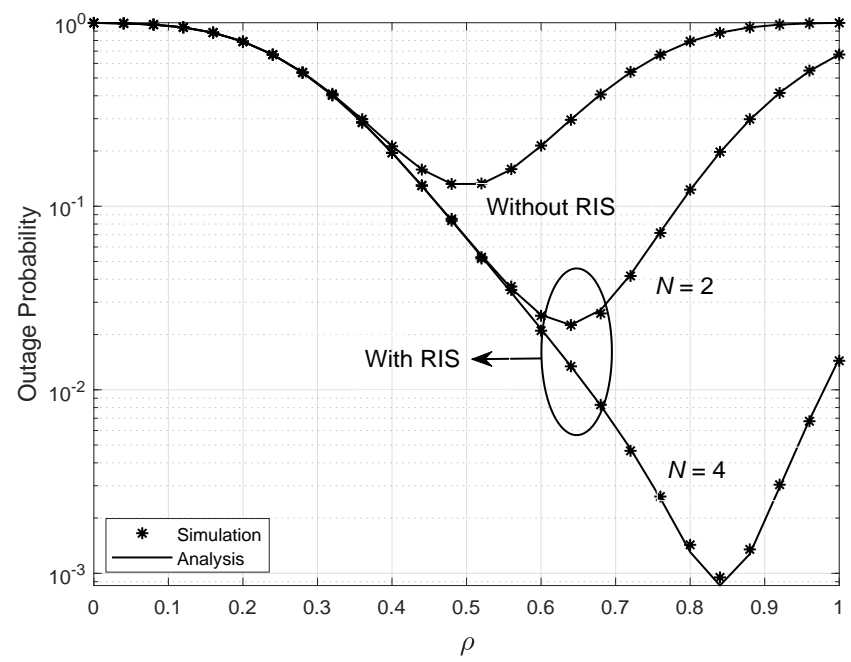

Fig. 3. Outage probability versus $\rho$ for dual-hop UAV systems with and without RISs

In Fig. 2, we plot the outage probability for our proposed system with and without RISs. It is clearly observed that the analytical results are in good agreement with the simulation results. Increasing $h$, the outage probability decreases first and then increases. The fundamental reason is that when $h$ is small, increasing $h$ can increase the possibility of the LoS transmission and in turn improves the link reliability. Continuing to increase $h$ results in a larger path loss and then the path loss dominates the system performance. Furthermore, under the same height, the outage probability of UAV communication systems assisted by RISs has been improved significantly.

In Fig. 3, we plot the outage probability curves versus $\rho$ for dual-hop UAV systems with and without RISs. From Fig. 3, the system outage probability decreases first and then increases as $\rho$ increases. As expected, we see that there is an optimal $\rho$ for a fixed $h$. The main reason is that the outage probability of the first hop is very small for small values of $\rho$ due to the use

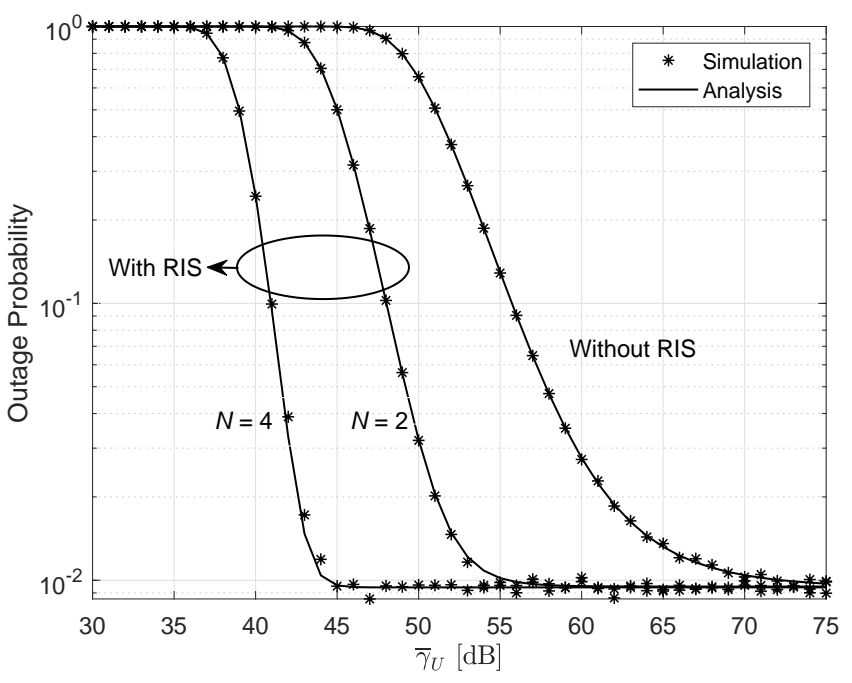

Fig. 4. Outage probability versus SNR for dual-hop UAV systems with and without RISs

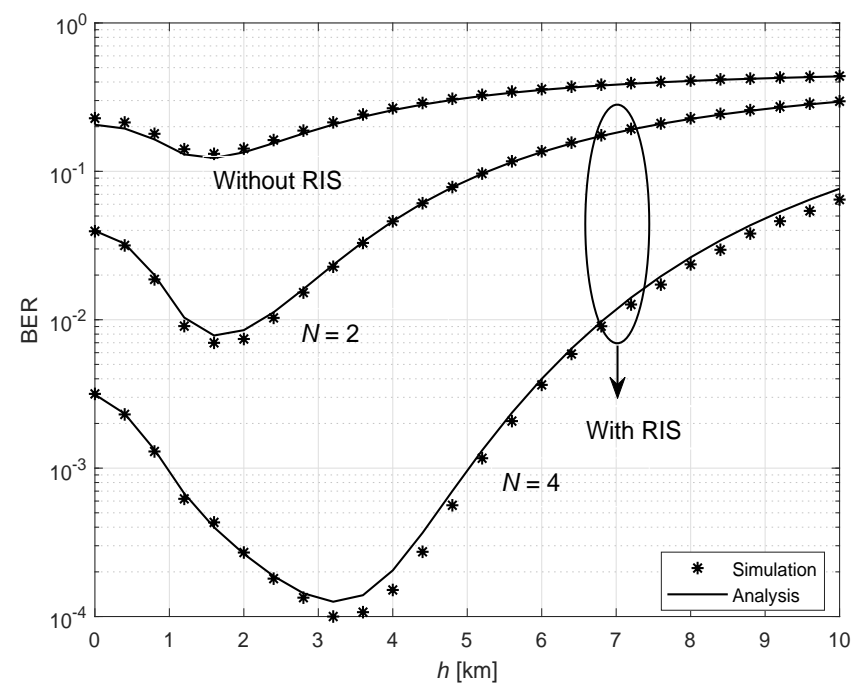

Fig. 5. Average BER versus $h$ for dual-hop UAV systems with and without RISs

of the RIS, and the outage performance is mainly determined by the second hop. Therefore, increasing $\rho$ means that the UAV becomes close to the destination and the path-loss in the second-hop becomes small, which results in a decreased outage probability. However, continuing to increase $\rho$, the firsthop would dominate the system performance. From Fig. 3, we can observe that the optimal position of the UAV for the system without the RIS is the middle location between T and D. But for the system with RISs, we can place the UAV close to $\mathrm{D}$ and then the best outage performance can be obtained.

In Fig. 4, we plot the outage probability curves versus $\bar{\gamma}_{U}$ for dual-hop UAV systems with and without RISs. As $\bar{\gamma}_{U}$ increases, the outage probability decreases. However, the outage probability tends to a constant for large values of $\bar{\gamma}_{U}$. Therefore, the system outage performance is mainly determined by the second link when RISs are used at the first link. Again, the outage probability of the system with RISs is 
obviously lower.

In Fig. 5, the average BER performance is presented when $p=1$ and $q=1$. As expected, the simulation and analytical results match very well. Similar to the observations in Fig. 2, the average BER first decreases and then increases when $h$ increases, and an optimal operating point can be found.

\section{Conclusions}

In this paper, an RIS-assisted UAV communication system with the DF protocol was proposed, and a $k$ - $\mu$ distribution was used to model the RIS-assisted G2A link. Based on this model, we analyzed the outage probability and average BER. The results show that RISs can significantly reduce the average BER, and improve the coverage and reliability of the proposed system. In particular, we can place the UAV close to the destination to obtain the best system performance when the RIS is applied.

\section{REFERENCES}

[1] L. Yang, J. Chen, M. O. Hasna, and H. Yang, "Outage performance of UAV-assisted relaying systems with RF energy harvesting," IEEE Commun. Lett., vol. 22, no. 12, pp. 2471-2474, Dec. 2018.

[2] J. Ye, C. Zhang, H. Lei, G. Pan, and Z. Ding, "Secure UAV-to-UAV systems with spatially random UAVs," IEEE Wireless Commun. Lett., vol. 8, no. 2, Apr. 2019

[3] H. Lei, D. Wang, K. Park, I. S. Ansari, G. Pan, and M. Alouini, "On secure UAV communication systems with randomly located eavesdroppers," 2019 IEEE/CIC International Conference on Communications in China (ICCC), Changchun, China, 2019, pp. 201-206.

[4] Y. Zeng, R. Zhang, and T. J. Lim, "Throughput maximization for UAVenabled mobile relaying systems," IEEE Trans. commun., vol. 64, no. 12, pp. 4983-4996, Dec. 2016.

[5] R. Marco Di, D. Merouane, P.-H. Dinh-Thuy, Z. Alessio, A. MohamedSlim, Y. Chau, S. Vincenzo, A. George C., H. Jakob, G. Haris, R. Julien de, B. Ahcene, L. Geoffroy, and F. Mathias, "Smart radio environments empowered by reconfigurable AI meta-surfaces: an idea whose time has come," EURASIP Journal on Wireless Communications and Networking., May 2019.

[6] S. Li, B. Duo, X. Yuan, Y.-C. Liang, and M. Di Renzo, "Reconfigurable intelligent surface assisted UAV communication: joint trajectory design and passive beamforming," Dec. 2019. [Online]. Available: arXiv: 1908.04082

[7] E. Basar, M. Di Renzo, J. De Rosny, M. Debbah, M. Alouini, and R. Zhang, "Wireless communications through reconfigurable intelligent surfaces," IEEE Access., vol. 7, pp. 116753-116773, 2019.

[8] C. Huang, G. C. Alexandropoulos, C. Yuen, and M. Debbah, "Indoor signal focusing with deep learning designed reconfigurable intelligent surfaces," 2019 IEEE 20th International Workshop on Signal Processing Advances in Wireless Communications (SPAWC)., Cannes, France, 2019, pp. 1-5.

[9] C. Huang, A. Zappone, G. C. Alexandropoulos, M. Debbah, and C. Yuen, "Reconfigurable intelligent surfaces for energy efficiency in wireless communication," IEEE Trans. Wireless Commun., vol. 18, no. 8, pp. 4157-4170, Aug. 2019.

[10] M. D. Yacoub, "The $k-\mu$ distribution and the $\eta-\mu$ distribution," IEEE Antenn Propag M, vol. 49, no. 1, pp. 68-81, Feb. 2007.

[11] J. C. S. Santos Filho, and M. D. Yacoub, "Highly accurate $k-\mu$ approximation to sum of $\mathrm{M}$ independent non-identical Ricean variates," Electron. Lett., vol. 41, no. 6, pp. 338-339, 17 Mar. 2005.

[12] M. M. Azari, F. Rosas, K. Chen, and S. Pollin, "Ultra reliable UAV communication using altitude and cooperation diversity," IEEE Trans. Commun., vol. 66, no. 1, pp. 330-344, Jan. 2018.

[13] A. Al-Hourani, S. Kandeepan, and S. Lardner, "Optimal lap altitude for maximum coverage," IEEE Wireless Commun. Lett., vol. 3, no. 6, pp. 569-572, 2014.

[14] S. Shimamoto et al., "Channel characterization and performance evaluation of mobile communication employing stratospheric platforms," IEICE transactions on communications., vol. 89, no. 3, pp. 937-944, 2006
[15] O. M. S. Al-Ebraheemy, A. M. Salhab, A. Chaaban, S. A. Zummo, and M. Alouini, "Precise performance analysis of dual-hop mixed RF/unifiedFSO DF relaying with heterodyne detection and two IM-DD channel models," in IEEE Photonics Journal., vol. 11, no. 1, pp. 1-22, Feb. 2019.

[16] I. S. Ansari, S. Al-Ahmadi, F. Yilmaz, M. Alouini and H. Yanikomeroglu, "A new formula for the BER of binary modulations with dual-branch selection over generalized-K composite fading channels," in IEEE Trans. Commun., vol. 59, no. 10, pp. 2654-2658, October 2011.

[17] P. C. Sofotasios, S. Muhaidat, G. K. Karagiannidis and B. S. Sharif, "Solutions to integrals involving the marcum $Q$-function and applications," IEEE Signal Process. Lett., vol. 22, no. 10, pp. 1752-1756, Oct. 2015. 